PROCEEDINGS OF THE

AMERICAN MATHEMATICAL SOCIETY

Volume 131, Number 10, Pages 3203-3210

S 0002-9939(03)06910-7

Article electronically published on May 9, 2003

\title{
THE SEMIGROUP GENERATED BY A SIMILARITY ORBIT OR A UNITARY ORBIT OF AN OPERATOR
}

\author{
C. K. FONG AND A. R. SOUROUR \\ (Communicated by David R. Larson)
}

\begin{abstract}
Let $T$ be an invertible operator that is not a scalar modulo the ideal of compact operators. We show that the multiplicative semigroup generated by the similarity orbit of $T$ is the group of all invertible operators. If, in addition, $T$ is a unitary operator, then the multiplicative semigroup generated by the unitary orbit of $T$ is the group of all unitary operators.
\end{abstract}

\section{INTRODUCTION}

Let $H$ be a separable infinite-dimensional complex Hilbert space and let $\mathcal{B}(H)$ be the algebra of all bounded operators on $H$. We consider the following question: What is the multiplicative semigroup generated by the similarity orbit of an invertible operator on $H$ ? An analogous question for the unitary group is: What is the multiplicative semigroup generated by the unitary orbit of a unitary operator?

Let us call a subset $\mathcal{S}$ of a group $\mathcal{G}$ conjugation invariant, or simply invariant, if $g^{-1} \mathcal{S} g \subseteq \mathcal{S}$ for every $g \in \mathcal{G}$. (An invariant group is also called a normal subgroup.) One may ask what are the invariant semigroups of the group $\mathcal{G} \mathcal{L}(H)$ of invertible operators, or, respectively, of the group $\mathcal{U}(H)$ of unitary operators.

We prove that if $T$ is an invertible operator that is not a scalar modulo the ideal $\mathcal{K}(H)$ of compact operators, then the multiplicative semigroup generated by the similarity orbit of $T$ is the group of all invertible operators. Consequently, every proper invariant semigroup in $\mathcal{G} \mathcal{L}(H)$ is included in $\mathbb{C} I+\mathcal{K}(H)$. This generalizes a theorem of Radjavi [11] that asserts that every invertible operator is a product of a finite number (seven) of involutions, and a theorem of the authors [7] that states that every invertible operator is a product of six unipotent operators.

Analogously, we show that if $U$ is a unitary operator that is not a scalar modulo the compacts, then the semigroup generated by the unitary orbit of $U$ is the group of all unitary operators. Consequently, every proper invariant semigroup in $\mathcal{U}(H)$ is included in $\mathbb{C} I+\mathcal{K}(H)$. This generalizes a theorem of Halmos and Kakutani 9]; namely, that every unitary operator is a product of four symmetries (i.e., selfadjoint unitary operators).

In the last section we prove a result about invariant groups in the Calkin algebra $\mathcal{B}(H) / \mathcal{K}(H)$.

Received by the editors November 22, 2000 and, in revised form, May 17, 2002.

2000 Mathematics Subject Classification. Primary 47D03; Secondary 20 F38.

Key words and phrases. Semigroups, conjugation-invariant.

This research was supported in part by an NSERC grant.

(C)2003 American Mathematical Society 
We end this introduction by noting that an additive version of the results in this paper is in [6]. A special case of the results in [6] is that every proper linear subspace of $\mathcal{B}(H)$ that is invariant under conjugation by all invertible operators (respectively, all unitary operators) is included in $\mathbb{C} I+\mathcal{K}(H)$. We also note that semigroups generated by a similarity orbit of a matrix have been investigated in [8].

\section{Statements of Results}

We start by stating the results about unitary operators.

Theorem A. Let $U$ be a unitary operator that is not the sum of a scalar and a compact operator. Then every unitary operator is a product of a finite number of operators each of which is unitarily equivalent to $U$.

The following is an immediate corollary.

Corollary 1. Every proper invariant semigroup (in particular, normal subgroup) in the group of unitary operators is included in $\mathbb{C} I+\mathcal{K}(H)$.

In Theorem A, if we take $U$ to be a symmetry (i.e., a unitary operator $U$ satisfying $\left.U^{2}=I\right)$, and if we also assume that both $\operatorname{ker}(U-I)$ and $\operatorname{ker}(U+I)$ are infinite dimensional, then we recover the qualitative part of the Halmos-Kakutani [9] result that states that every unitary operator is a product of four symmetries.

The theorem of Halmos and Kakutani has a "skew" version due to Radjavi 11]; namely, that every invertible operator is a product of seven involutions. (An involution is an operator whose square is the identity.) We also have the following "skew" version of Theorem A

Theorem B. Let $T$ be an invertible operator which is not the sum of a scalar and a compact operator. Then every invertible operator is a product of a finite number of operators each of which is similar to $T$.

As before, we conclude the following about invariant semigroups.

Corollary 2. Every proper invariant semigroup (in particular, normal subgroup) in the group of invertible operators is included in $\mathbb{C} I+\mathcal{K}(H)$.

The following are special cases of Theorem B First recall that an operator is said to be unipotent if it is the sum of the identity and a nilpotent operator, and is said to be a unipotent of order 2 if it is of the form $I+N$, where $N^{2}=0$.

Corollary 3. Every invertible operator is a product of a finite number of

(a) involutions ( $c f$. [11);

(b) unipotents of order 2 (cf. [7]);

(c) invertible positive operators (cf. [10]).

We again observe that in [11], [7] and [10], the number of factors are seven, six and seven, respectively. See also [13].

Proof. Parts (a) and (b) are obvious. To prove part (c), let $P$ be an invertible positive operator that is not a scalar plus compact. By Theorem $B$, every invertible operator is a product of a finite number of operators each of which is similar to $P$. Each factor $S^{-1} P S$ is a product of two invertible positive operators since $S^{-1} P S=S^{-1}\left(S^{-1}\right)^{*}\left(S^{*} P S\right)$. 
We end this section with the following remarks about the number of factors in Theorems $\mathrm{A}$ and $\mathrm{B}$.

Remarks. The number of factors in Theorem $\mathrm{A}$ is unbounded. Indeed, if $U$ is a unitary operator satisfying $\|U-I\| \leq 2^{-n}$, and if $V$ is a product of $n$ operators from the unitary orbit of $U$, then it is easy to see that $\|V-I\| \leq 1$. On the other hand, the proof of Theorem $\mathrm{B}$ given below establishes that 112 factors suffice for the factorization of that theorem. This is undoubtedly not a sharp estimate, but we make no attempt in the present work to investigate the minimum number of factors required.

\section{Proof of Theorem $\mathrm{A}$}

We denote the essential numerical range of an operator $A$ by $W_{e}(A)$. For basic properties of the essential numerical range, the reader is referred to [5].

Lemma 1. If $U$ is a unitary operator and if zero is in the interior of the numerical range of $U$, then every unitary operator is a product of at most eight operators each of which is unitarily equivalent to $U$.

Proof. We denote the interior of the numerical range of $U$ by $W_{e}(U)^{o}$. Construct inductively an orthonormal sequence $\left\{e_{n}\right\}$ such that $\left(U e_{n}, e_{m}\right)=0$ for all $n, m$, as follows. Since $0 \in W_{e}(U)^{o} \subseteq W(U)$, there is a unit vector $e_{1}$ such that $\left(U e_{1}, e_{1}\right)=$ 0 . Suppose now that we already have $e_{1}, \ldots, e_{k}$ such that $\left(U e_{n}, e_{m}\right)=0$ for all $n, m \leq k$. Let

$$
\mathcal{M}=\left\{e_{1}, \ldots, e_{k}, U e_{1}, \ldots, U e_{k}, U^{*} e_{1}, \ldots, U^{*} e_{k}\right\}^{\perp}
$$

and let $V$ be the compression of $U$ to $\mathcal{M}$. Since $\mathcal{M}^{\perp}$ is finite dimensional, we have $W_{e}(V)=W_{e}(U)$ and hence $0 \in W_{e}(V)^{o}$. Let $e_{k+1}$ be a unit vector in $\mathcal{M}$ such that $\left(V e_{k+1}, e_{k+1}\right)=0$. Then $e_{1}, \ldots, e_{k+1}$ is a finite orthonormal sequence such that $\left(U e_{n}, e_{m}\right)=0$ for all $n, m \leq k+1$.

Let $H_{1}$ be the closed linear span of $\left\{e_{n}: n\right.$ odd $\}$, let $H_{3}=U H_{1}$ and let $H_{2}=$ $\left(H_{1} \oplus H_{3}\right)^{\perp}$. The unitary operator $U$ maps $H_{1}$ onto $H_{3}$ and hence it maps $H_{2} \oplus H_{3}=$ $H_{1}^{\perp}$ onto $H_{3}^{\perp}=H_{1} \oplus H_{2}$ and so the matrix of $U$ relative to the decomposition $H=H_{1} \oplus H_{2} \oplus H_{3}$ takes the form

$$
U=\left(\begin{array}{ccc}
0 & * & * \\
0 & * & * \\
R & 0 & 0
\end{array}\right)
$$

where $R$ is a unitary operator from $H_{1}$ onto $H_{3}$. We note that each of $H_{1}, H_{2}$ and $\mathrm{H}_{3}$ is isomorphic to $H$.

Now let $V$ be any unitary operator on $H_{3}$ and let

$$
V_{0}=\left(\begin{array}{ccc}
0 & 0 & R^{*} V \\
0 & 1 & 0 \\
R & 0 & 0
\end{array}\right)
$$

Then $V_{0}$ is a unitary operator on $H$ and

$$
U V_{0} U V_{0}^{*}=\left(\begin{array}{ccc}
* & * & 0 \\
* & * & 0 \\
0 & 0 & V
\end{array}\right)=\left(\begin{array}{cc}
V^{\prime} & 0 \\
0 & V
\end{array}\right)
$$


Identifying each of $H_{1}+H_{2}$ and $H_{3}$ with $H$, the above computation shows that if $V$ is a unitary operator on $H$, then there exists another unitary operator $V^{\prime}$ such that $V \oplus V^{\prime}$ is a product of two operators unitarily equivalent to $U$. We now take $V$ to be a bilateral shift of infinite multiplicity. The unitary operators $V^{\prime}$ can be written as a product $V_{1} V_{2}$ of two bilateral shifts of infinite multiplicity [9]. Let $J$ be a unitary operator such that $V^{*}=J V_{1} J^{*}$ and let $S=J V_{2} J^{*}$. It follows that $V \oplus V^{\prime}$ is unitarily equivalent to $V \oplus V^{*} S$ and so each of $V \oplus V^{*} S$ and $V^{*} S \oplus V$ is a product of two operators unitarily equivalent to $U$. So, there exist four operators unitarily equivalent to $U$ whose product is the operator $\left(V \oplus V^{*} S\right)\left(V^{*} S \oplus V\right)=S \oplus V^{*} S V$ which is a bilateral shift of infinite multiplicity. Now the conclusion of the lemma follows by using, once again, the fact that every unitary operator is a product of two bilateral shifts of infinite multiplicity.

Proof of Theorem $\AA$. Suppose that $U$ is a unitary operator which is not a scalar plus compact. The essential spectrum $\sigma_{e}(U)$ of $U$ contains two distinct complex numbers $\lambda_{1}$ and $\lambda_{2}$. We may write $U$ in the form

$$
U=\left(\begin{array}{ccc}
\lambda_{1} 1 & 0 & 0 \\
0 & \lambda_{2} 1 & 0 \\
0 & 0 & A
\end{array}\right)+K_{1}
$$

where $K_{1}$ is a compact operator and where every direct summand is infinite dimensional (see, e.g., [5, Theorem 4.2]). In view of Lemma 1] it suffices to show that there is a product $V$ of a finite number of operators unitarily equivalent to $U$ such that $0 \in W_{e}(V)^{o}$.

We consider two cases according as $\lambda_{2}=-\lambda_{1}$ or not. In the first case,

$$
U=\lambda_{1}\left(\begin{array}{ccc}
1 & 0 & 0 \\
0 & -1 & 0 \\
0 & 0 & B
\end{array}\right)+K_{1}
$$

which is unitarily equivalent to

$$
\lambda_{1}\left(\begin{array}{ccc}
0 & J & 0 \\
J^{*} & 0 & 0 \\
0 & 0 & B
\end{array}\right)+K_{1}
$$

for every unitary operator $J$. Now let $R$ be a unitary operator such that $0 \in W_{e}(R)^{o}$. It follows that $U$ is unitarily equivalent to each of the operators

$$
U_{1}=\lambda_{1}\left(\begin{array}{ccc}
0 & R & 0 \\
R^{*} & 0 & 0 \\
0 & 0 & B
\end{array}\right)+K_{1}
$$

and

$$
U_{2}=\lambda_{1}\left(\begin{array}{lll}
0 & 1 & 0 \\
1 & 0 & 0 \\
0 & 0 & B
\end{array}\right)+K_{1}
$$

hence

$$
U_{1} U_{2}=\lambda_{1}^{2}\left(\begin{array}{ccc}
R & 0 & 0 \\
0 & R^{*} & 0 \\
0 & 0 & B^{2}
\end{array}\right)+K_{2}
$$

where $K_{2}$ is compact. Therefore $0 \in W_{e}\left(U_{1} U_{2}\right)^{o}$. This ends the proof in this case. 
Finally, we consider the case $\lambda_{2} \neq-\lambda_{1}$. Let $\mu=\lambda_{2} / \lambda_{1}$, so $\mu \neq \pm 1$. It is easy to see that there exists a positive integer $n$ such that 0 belongs to the interior of the convex hull of $\left\{1, \mu, \mu^{2}, \ldots, \mu^{n}\right\}$. For every positive integer $m$, we have

$$
U^{m}=\lambda_{1}^{m}\left(\begin{array}{ccc}
1 & 0 & 0 \\
0 & \mu^{m} & 0 \\
0 & 0 & B^{m}
\end{array}\right)+K_{m}
$$

where $K_{m}$ is compact. So $U^{m}$ is unitarily equivalent to the operator

$$
V_{m}=\lambda_{1}^{m} \operatorname{diag}\left(1, \ldots, 1, \mu^{m}, 1, \ldots, 1, B^{m}\right)+K_{m}
$$

with $n+2$ direct summands and with $\mu^{m}$ in the $(m+1)$ st position. Now let $V=V_{1} V_{2} \cdots V_{n}$, so

$$
V=\lambda \operatorname{diag}\left(1, \mu, \mu^{2}, \ldots, \mu^{n}, C\right)+K
$$

for a unimodular complex number $\lambda$, a bounded operator $C$ and a compact operator $K$. Therefore $0 \in W_{e}(V)^{o}$ and $V$ is a product of $n(n+1) / 2$ operators that are unitarily equivalent to $U$.

\section{Proof of Theorem B}

We begin by stating a well-known result (see [12, Cor. 0.15]). Recall that $\sigma(A)$ denotes the spectrum of an operator $A$.

Lemma 2. If $\sigma(A) \cap \sigma(B)=\varnothing$, then the operator $\left(\begin{array}{cc}A & 0 \\ B\end{array}\right)$ is similar to $A \oplus B$.

To prove Theorem $\mathrm{B}$, assume that $T$ is an invertible operator which is not a scalar modulo the compacts. By a result of Brown and Pearcy [1, Theorem 2], $T$ is similar to an operator of the form

$$
T_{0}=\left(\begin{array}{lll}
0 & A & B \\
0 & C & D \\
1 & E & F
\end{array}\right)
$$

acting on $H \oplus H \oplus H$. Let $S$ be an arbitrary invertible operator, let

$$
L_{1}=\left(\begin{array}{ccc}
0 & 0 & 1 \\
0 & 1 & 0 \\
1 & 0 & 0
\end{array}\right), \quad L_{2}=\left(\begin{array}{ccc}
S & 0 & 0 \\
0 & 1 & 0 \\
0 & 0 & 1
\end{array}\right),
$$

and let $T_{j}=L_{j}^{-1} T_{0} L_{j}$ for $j=1,2$. Then each of $T_{1}$ and $T_{2}$ is similar to $T$ and

$$
T_{2} T_{1}=\left[\begin{array}{cc}
F(S) & 0 \\
* & S
\end{array}\right]
$$

where

$$
F(S)=\left[\begin{array}{cc}
S^{-1} & 0 \\
0 & 1
\end{array}\right]\left[\begin{array}{cc}
V A & V B \\
C & D
\end{array}\right]\left[\begin{array}{cc}
D & C \\
V B & V A
\end{array}\right]
$$

For every invertible operator $X$, we will show that $\sigma(\alpha X) \cap \sigma(F(\alpha X))=\varnothing$ if $|\alpha|$ is either large enough or small enough. To prove this, notice that $F(\alpha X)=$ $\left[\begin{array}{cc}\alpha^{-1} & 0 \\ 0 & 1\end{array}\right] F(X)$, so $\|F(\alpha X)\| \leq\|F(X)\|$ for $|\alpha| \geq 1$ and hence we can choose $|\alpha|$ large enough so that $\sigma(\alpha X)$ lies outside the disc $\{z:|z| \leq\|F(X)\|\}$ which includes $\sigma(F(\alpha X))$. Similarly, for $|\alpha|$ small enough, $\sigma(\alpha X)$ is included in the disc $\{z:|z|<$ $\left.\left\|F(X)^{-1}\right\|^{-1}\right\}$, while $\sigma(F(\alpha X))$ lies outside the same disc since $\left\|F(\alpha X)^{-1}\right\| \leq$ 
$\left\|F(X)^{-1}\right\|$ for $|\alpha| \leq 1$. Applying the above to $X=S$ and $X=1$ and using Lemma 2, we conclude that there exists a scalar $\alpha$ such that each of the operators

$$
\left[\begin{array}{cc}
F(\alpha S) & 0 \\
0 & \alpha S
\end{array}\right] \text { and }\left[\begin{array}{cc}
F\left(\alpha^{-1} 1\right) & 0 \\
0 & \alpha^{-1} 1
\end{array}\right]
$$

is a product of two operators similar to $T$, and so $S \oplus F(\alpha S) F\left(\alpha^{-1} 1\right)$ is a product of four operators similar to $T$.

Now take $S$ to be $U \oplus 1$ where $U$ is a bilateral shift with infinite multiplicity and 1 is the identity operator on an infinite dimensional space. From the above, there exists an invertible operator $Q$ on $H$ such that $S \oplus Q$ is a product of four operators similar to $T$. The operator $S \oplus Q$ can be written as $U \oplus Q^{\prime}$ where both $U$ and $Q^{\prime}$ are operators on $\sum_{n \in Z} \oplus H_{n}$ with $H_{n}=H_{0}$ for all $n$ and

$$
\begin{gathered}
U\left(\ldots, x_{-2}, x_{-1}, x_{0}, x_{1}, \ldots\right)=\left(\ldots, x_{-2}, x_{-1}, x_{0}, x_{1}, \ldots\right), \\
Q^{\prime}=\operatorname{diag}(\ldots, 1, Q, 1,1, \ldots)
\end{gathered}
$$

that is,

$$
Q^{\prime}\left(\ldots, x_{-2}, x_{-1}, x_{0}, x_{1}, x_{2}, \ldots\right)=\left(\ldots, x_{-2}, x_{-1}, Q x_{0}, x_{1}, x_{2}, \ldots\right) .
$$

(The box $\square$ is used to indicate the zero ${ }^{\text {th }}$ position.) Now

$$
\begin{gathered}
\left(U \oplus Q^{\prime}\right)\left(Q^{\prime} \oplus U\right)=U Q^{\prime} \oplus Q^{\prime} U \\
U Q^{\prime}\left(\ldots, x_{-2}, x_{-1}, x_{0}, x_{1}, x_{2}, \ldots\right)=\left(\ldots, x_{-2}, x_{-1}, Q x_{0}, x_{1}, x_{2}, \ldots\right) .
\end{gathered}
$$

Let $J=\operatorname{diag}(\ldots, 1,1,1, Q, Q, \ldots)$. By direct computation, it follows that $J\left(U Q^{\prime}\right) J^{-1}=U$. In the same way, we can show that $Q^{\prime} U$ is similar to $U$. Therefore $\left(U \oplus Q^{\prime}\right)\left(Q^{\prime} \oplus U\right)$ is similar to a bilateral shift of infinite multiplicity. We have shown that a bilateral shift is a product of eight operators similar to $T$. Since each symmetry is a product of two bilateral shifts of infinite multiplicity, the theorem follows from Radjavi's result [11] which asserts that every invertible operator is a product of at most seven involutions.

\section{Groups in the Calkin algebra}

The Calkin algebra $\mathcal{B}(H) / \mathcal{K}(H)$ will be denoted by $\mathfrak{A}$. The group of invertible elements and unitary elements of $\mathfrak{A}$ will be denoted by $G L(\mathfrak{A})$ and $U(\mathfrak{A})$, respectively. In this section, we make a few remarks about semigroups generated by a conjugacy class in $G L(\mathfrak{A})$ and $U(\mathfrak{A})$. Recall that two elements $a$ and $b$ of a group $\mathcal{G}$ are said to be conjugate if $a=g^{-1} b g$ for some $g \in \mathcal{G}$.

Before proceeding, we recall some facts about the Calkin algebra and index theory (see 4, Chapter 5]). The index of a Fredholm operator $T$ is defined by $\operatorname{ind}(T)=\operatorname{dim} \operatorname{ker}(T)-\operatorname{dim} \operatorname{ker}\left(T^{*}\right)$. The index satisfies the equation $\operatorname{ind}(T S)=$ $\operatorname{ind}(T)+\operatorname{ind}(S)$. Furthermore, it is invariant under compact perturbations. Let $\pi: \mathcal{B}(H) \rightarrow \mathfrak{A}$ be the canonical quotient map. Atkinson's theorem 4, Theorem 5.17 implies that the set of Fredholm operators is the inverse image under $\pi$ of the set $G L(\mathfrak{A})$ of invertible operators in $\mathfrak{A}$. In view of this and the invariance of the index under compact perturbations, we define the index of an invertible element in $\mathfrak{A}$ by $\operatorname{ind}(a)=\operatorname{ind}(A)$ for any $A \in \pi^{-1}(a)$. This gives a homomorphism from the group $G L(\mathfrak{A})$ onto the group of integers $\mathbb{Z}$. 
Two facts about operators of index 0 are needed in the sequel.

(1) For a Fredholm operator $T$, $\operatorname{ind}(T)=0$ if and only if $T$ is a compact perturbation of an invertible operator.

(2) If $\pi(T)$ is unitary and if $\operatorname{ind}(T)=0$, then $T$ is a compact perturbation of a unitary operator [2, Theorem 3.1].

One more fact about the Calkin algebra $\mathfrak{A}$ is that the centre of $\mathfrak{A}$ is the scalars [3]. It follows immediately that the centre of the group $G L(\mathfrak{A})$ is also the (nonzero) scalars. We can also easily establish the fact that the centre of the group $U(\mathfrak{A})$ is $\{\lambda 1:|\lambda|=1\}$ since every element of $\mathfrak{A}$ is a linear combination of four unitary elements. (Indeed, if $a$ is self-adjoint with $\|a\| \leq 1$, then $a \pm\left(1-a^{2}\right)^{1 / 2}$ are unitaries, and hence $a$ is a convex combination of two unitaries.)

We now state two immediate consequences of Theorems $\mathrm{A}$ and $\mathrm{B}$.

Proposition 1. Let a be an invertible (respectively, a unitary) element of $\mathfrak{A}$ of index 0 . If a is not a scalar, then the semigroup generated by the conjugacy class of a in $G L(\mathfrak{A})$ (respectively, $U(\mathfrak{A})$ ) is the subgroup of all elements of index 0.

Proof. Since $\operatorname{ind}(a)=0$, there exists an invertible operator $B$ such that $\pi(B)=b$. Furthermore, if $a$ is a unitary, then the operator $B$ may be chosen to be a unitary [2, Theorem 3.1]. Since $B$ is not a scalar modulo the compacts, the results follow from Theorems $\mathrm{A}$ and $\mathrm{B}$

For more general elements, we consider only the group generated by the conjugacy class. First, we need a lemma.

Lemma 3. If a is an invertible element in the Calkin algebra $\mathfrak{A}$ such that $a^{-1} u^{-1}$ au is a scalar for every unitary element $u$ in $\mathfrak{A}$, then a is a scalar.

Proof. Let $b$ be a self-adjoint element in $\mathfrak{A}$. Since $e^{i t b}$ is unitary for every real number $t$, there exist scalars $\lambda_{t}$ such that $a^{-1} e^{-i t b} a e^{i t b}=\lambda_{t} 1$ for every scalar $t$. Taking the derivative at $t=0$, we get that $b-a^{-1} b a=\lambda 1$ for a scalar $\lambda$. Thus

$$
\sigma(b)=\sigma\left(a^{-1} b a\right)=\sigma(b-\lambda 1)=\sigma(b)-\lambda .
$$

This implies that $\lambda=0$ and hence $a b=b a$; i.e., $a$ commutes with every self-adjoint element in $\mathfrak{A}$. It follows that $a$ commutes with every element in $\mathfrak{A}$ and so $a$ is a scalar.

Proposition 2. Let $\mathcal{G}$ be either the group $G L(\mathfrak{A})$ of all invertible elements or the group $U(\mathfrak{A})$ of all unitary elements in the Calkin algebra. If a is an element of $\mathcal{G}$ with a nonzero index $n$, then the group generated by the conjugacy class of a in $\mathcal{G}$ is $\{g \in \mathcal{G}: n$ divides ind $(g)\}$.

Proof. Let $\mathcal{N}$ be the group generated by the conjugacy class of $a$. Since $a$ is not a scalar, it follows from Lemma 3 that there exists a unitary element $u$ in $\mathfrak{A}$ such that $b:=a^{-1} u^{-1} a u$ is not a scalar. Now $b \in \mathcal{N}$ and ind $(b)=0$. By Proposition [1] we have that $\mathcal{N} \supseteq \mathcal{G}_{0}:=\{g \in \mathcal{G}: \operatorname{ind}(g)=0\}$. Since $\mathcal{G}_{0}$ is the kernel of the homomorphism ind: $\mathcal{G} \rightarrow \mathbb{Z}$, the subgroup $\mathcal{N}$ is the inverse image under the index map of a subgroup of $\mathbb{Z}$, and the result follows.

Corollary 4. Let $\mathcal{G}$ be either the group $G L(\mathfrak{A})$ of all invertible elements or the group $U(\mathfrak{A})$ of all unitary elements in the Calkin algebra. Every normal subgroup of $\mathcal{G}$ is either included in the centre (i.e., the scalars) or is $\{g \in \mathcal{G}: n$ divides $\operatorname{ind}(g)\}$, for some integer $n$. 
Corollary 5. Let $\mathcal{G}$ be as above, and let $S$ be the unilateral shift and $\mathbf{s}$ its image in the Calkin algebra. Then the group generated by the conjugacy class of $\mathbf{s}$ in $\mathcal{G}$ is all of $\mathcal{G}$.

\section{REFERENCES}

[1] A. Brown and C. Pearcy, Structure of commutators of operators, Ann. Math. 82 (1965), 112-127. MR 31:2612

[2] L. G. Brown, R. G. Douglas and P. A. Fillmore, Unitary equivalence modulo the compact operators and extensions of $C^{*}$-algebras, pp. 58-128, Lecture Notes in Mathematics, Vol. 345, 1973, Springer-Verlag, Berlin. MR 52:1378

[3] J. W. Calkin, Two-sided ideals and congruences in the ring of bounded operators in Hilbert space, Ann. Math. (2) 42 (1941), 839-873. MR 3:208c

[4] R. G. Douglas, Banach algebra techniques in operator theory, Academic Press, New York, 1972. 50:14335

[5] P. A. Fillmore, J. G. Stampfli and J. P. Williams, On the essential numerical range, the essential spectrum and a problem of Halmos, Acta. Sci. Math. (Szeged) 33 (1972), 179-192. MR 48:896

[6] C. K. Fong, C. R. Miers and A. R. Sourour, Lie and Jordan ideals of operators on Hilbert space, Proc. Amer. Math. Soc. 84 (1982), 516-520. MR 84g:47039

[7] C. K. Fong and A. R. Sourour, The group generated by unipotent operators, Proc. Amer. Math. Soc. 97 (1986), 453-458. MR 87m:47099

[8] L. Grunenfelder, M. Omladic, H. Radjavi and A. R. Sourour, Semigroups generated by similarity orbits, Semigroup Forum 62 (2001), 460-472. MR 2002g:20107

[9] P. R. Halmos and S. Kakutani, Products of symmetries, Bull. Amer. Math. Soc. 64 (1958), 77-78. MR 20:6658

[10] N. C. Phillips, Every invertible Hilbert space operator is a product of seven positive operators, Canad. Math. Bull. 38 (1995), 230-236. MR 96h:47044

[11] H. Radjavi, The group generated by involutions, Proc. Roy. Irish. Acad., Section A, 81 (1981), 9-12. MR 83c: 47006

[12] H. Radjavi and P. Rosenthal, Invariant subspaces, Springer-Verlag, Berlin, 1973. MR 51:3924

[13] P. Y. Wu, The operator factorization problems, Linear Algebra Appl. 117 (1989), 35-63. MR 90g:47031

School of Mathematics and Statistics, Carleton University, Ottawa, Ontario, Canada K1S 5B6

Department of Mathematics and Statistics, University of Victoria, Victoria, British Columbia, Canada V8W 3P4

E-mail address: sourour@math.uvic.ca 